\title{
Relation between left ventricular filling pressure and angiographic findings in coronary heart disease Ventriculography used as a stress test
}

\author{
K. FORFANG, A. ANDERSEN, S. SIMONSEN, AND G. STAKE \\ From Cardiological Laboratory, Medical Department B, and Department of Radiology, \\ University Hospital, Rikshospitalet, Oslo, Norway
}

In 80 male patients with coronary heart disease maximum diastolic pressure in the left ventricle (LVMDP) (usually the top of the a wave), and 'post a' end-diastolic pressure (LVEDP) before and 2 and 3 minutes after ventriculography were correlated to angiographic estimates of left ventricular function (aneurysm, ejection fraction (EF), and to lesions shown by selective coronary arteriography using a score system (coronary artery lesions index, CALI).

A significant correlation between CALI and LVEDP (or LVMDP) could not be shown either before or after ventriculography. Before ventriculography, however, LVEDP and LVMDP were good predictors of left ventricular dyskinesia (aneurysm and/or $E F<50 \%$ ). A positive and significant correlation between $C A L I$ and the LVEDP (and LVMDP) increments following ventriculography ( $\triangle L V E D P, \triangle L V M D P$ ) was found in patients with LVEDP (or LVMDP) below $12 \mathrm{mmHg}$ before ventriculography. Using $\triangle L V E D P$ the correlation coefficient was $0.51(n=41, P<0.001$, 95 per cent confidence interval 0.24 to 0.88$)$. Using $\triangle$ LVMDP $r=0.47(n=41,0.001<P<0.01) . \Delta L V E D P>12 \mathrm{mmHg}$ was found only in patients with triple vessel disease.

In coronary artery disease a prominent $a$ wave in the left ventricular pressure curve is a frequent finding. This is assumed to be a result of reduced ventricular compliance which is often found in these conditions (Bristow, Van Zee, and Judkins, 1970; Diamond and Forrester, 1972; Smith et al., 1974). Stresstests, as exercise or injection of contrast medium into the left ventricle, often lead to increased filling pressure (Goldschlager et al., 1970; Cohn et al., 1973), and it is shown that these pressure increments are partly related to distribution and severity of coronary artery lesions (Goldschlager et al., 1970; Brundage and Cheitlin, 1971; Saltups et al., 1971). In some patients the $a$ wave increments after ventriculography are very striking, yet may not be accompanied by rises in end-diastolic pressure (post a). The aim of this work was to study the relation between the angiographic findings and the left ventricular filling pressure before and after ventriculography, using pressures measured at the top of the $a$ wave as well as 'post $a$ ' end-diastolic pressures, both in patients with normal left ven-

Received for publication 9 April 1976 tricular contraction, and in cases with different degrees of dyskinesia.

\section{Subjects and methods}

Eighty male patients in hospital for coronary heart disease were studied. The ages ranged from 28 to 69 years, mean 50.4 (SD 8.3). All of them had a characteristic history of angina pectoris, and 59 had previous myocardial infarction. The duration of the symptoms ranged from 2 months to 14 years: in 55 of the patients the symptoms had persisted for more than 2 years. All patients were in sinus rhythm. Retrograde catheterisation of the left ventricle and selective coronary angiography were done using the following procedure. The ventricle was catheterised via the left femoral artery using a Pigtail Ducor $8 \mathrm{~F}$ catheter. After pressure recordings ventriculography was done with the patient in the right anterior oblique (RAO) position using $35 \mathrm{~mm}$ film at 75 frames per second. Isopaque Coronar (metrizoate meglumine/ $\mathrm{Na} / \mathrm{Ca}$ (58/9/1), $370 \mathrm{mg} \mathrm{J} / \mathrm{ml}) 45 \mathrm{ml}$, was injected by a pressure injector (Gidlund) $\left(8 \mathrm{~kg} / \mathrm{cm}^{2}\right)$. 


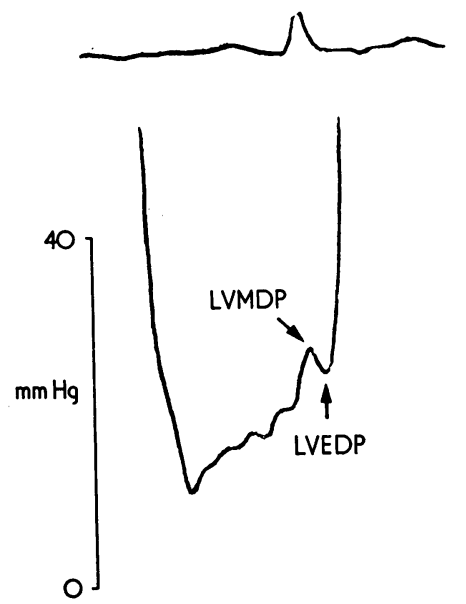

Fig. 1 The diastolic part of a pressure recording from the left ventricle showing a prominent ' $a$ ' wave. The arrows indicate where LVMDP and LVEDP were measured.

In cases with suspected ventricular aneurysm, an additional injection was made in the left anterior oblique (LAO) position. Two to three minutes after the first contrast injection a new set of left ventricular pressures was recorded. A further angiogram was recorded with the catheter in the aortic root. Selective coronary angiography was by the method described by Judkins (1967).

The pressure recordings were made using ElemaSchönander pressure transducer, EMT 35, No. 2359, with zero reference level on the anterior axillary line in the fourth intercostal space. The left ventricular pressures measured, taking the mean pressures of 10 successive cycles, were peak systolic pressure, maximum diastolic pressure (LVMDP) (often at the top of the $a$ wave), and end-diastolic pressure (LVEDP) (Fig. 1).

LVEDP was measured after the $a$ wave just before the rapid systolic upstroke. Sometimes LVMDP and LVEDP were equal, either because the $a$ wave could not be clearly identified, or because the top of the $a$ wave equalled LVEDP. The differences in LVEDP and LVMDP obtained before and after ventriculography ( $\triangle$ LVEDP, $\triangle$ LVMDP) were measured for correlation studies (Fig. 2). The heart rates before and after ventriculography were also noted, and rate-pressure products (heart rate $\times$ peak systolic pressure) were calculated.

Left ventricular movements and contractility were estimated by eyeball appreciation and ejection fraction (EF) measured by planimetry using the ellipsoidal formulae for calculation described by Arbogast, Solignac, and Bourassa (1973). Based

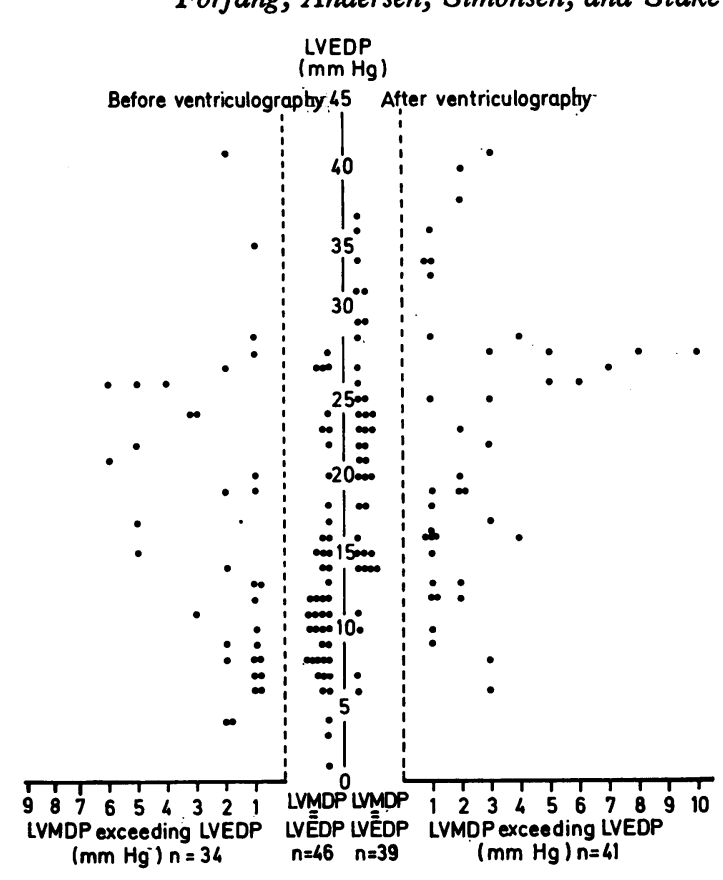

Fig. 2 Relation between LVMDP and LVEDP before (left) and after ventriculography (right). Cases where LVMDP and LVEDP were equal are plotted between the dotted lines. Cases where LVMDP exceeded LVEDP are plotted lateral to the dotted lines according to LVEDP (ordinate, centre line), and the difference between LVMDP and LVEDP ( $\mathrm{mmHg}$ ) along the abscissa.

on these observations the patients were divided in 3 groups.

Group 1: EF 50 per cent or more. No signs of left ventricular aneurysm.

Group 2: EF below 50 per cent. No signs of left ventricular aneurysm.

Group 3: Aneurysm of the left ventricular wall with bulging contour and paradoxical movements.

The coronary artery lesions were evaluated by a score system, where the relative importance of the arterial lesions for the myocardial blood flow was considered. The three main arteries (right coronary artery, left anterior descending artery, and left circumflex artery) were studied separately and given a score ranging from 0 to 4 . The degree of obstruction was estimated for the main artery and its greater branches. Lesions of the main stem of the left coronary artery were given a double score.

Score 0: Artery without significant stenosis (i.e. $<25 \%$ obstruction).

Score 1: 25 to 50 per cent obstruction of the proximal lumen, or lesions in the distal parts considered to reduce the total vessel lumen less than 50 per cent. 
Score 2:50 to 75 per cent obstruction of the proximal lumen, or lesions in the distal parts considered to reduce the total vessel lumen equally.

Score 3: 75 to 95 per cent obstruction of the proximal lumen, or lesions in the distal parts considered to reduce the total vessel lumen equally.

Score 4: Occlusion before origin of greater branches.

Coronary artery lesions index (CALI) is the sum of the scores of the individual arteries (maximum score 12).

The angiograms were examined by all of us without knowing the pressure data, and the assessments were made by a joint decision.

The statistical methods used were linear correlation analysis, and Student's t-test, paired and unpaired.

\section{Results}

\section{ANGIOGRAPHY}

The grouping of the patients according to the ventriculography gave the following results: group 1 $(\mathrm{EF} \geq 50 \%): 54$ patients; group 2 (EF $<50 \%)$ : 13 patients; group 3 (left ventricular aneurysm): 14 patients. In group 2 the EF ranged from 5 to 38 per cent, mean $23 \cdot 3$, SD $9 \cdot 8$.

A graphic presentation of the material according to CALI (range 0 to 11 ) is shown in Fig. 3 to 6. The patients were also divided in groups according to single-, double-, and triple-vessel disease. The numbers of cases in these groups were 7,20 , and 50 respectively. In 3 cases there were angiographically normal arteries.

\section{PRESSURES}

Both peak systolic ventricular pressure and heart rate increased after ventriculography; mean pressure from $122 \mathrm{mmHg}$ to $129(\mathrm{P}<0.001)$, and rate from 78 to $86(P<0.001)$. Mean rate-pressure product increased from 9571 before to 11081 after ventriculography $(P<0.001)$.

LVMDP and LVEDP before and after ventriculography are shown in Fig. 2. In nearly half of the patients LVMDP exceeded LVEDP. Before ventriculography the mean difference between LVMDP and LVEDP was $0.9 \mathrm{mmHg}$ as compared with 1.2 after ventriculography $(P>0.05)$. The differences were most prominent in cases with LVEDP between 15 and $30 \mathrm{mmHg}$.

\section{CORRELATIONS BETWEEN ANGIOGRAPHY}

AND PRESSURES

The relation between LVEDP before ventriculography and angiographic findings is shown in Fig. 3. There was no correlation between LVEDP and CALI. Raised LVEDP, however, was a more frequent finding in the patients belonging to the dyskinesia groups 2 and 3 as compared with patients with EF $\geq 50$ per cent. LVEDP above $16 \mathrm{mmHg}$ was found in 9 of the 12 patients in group 2, and in 10 of the 14 patients in group 3. In the patients with $E F \geq 50$ per cent (group 1), however, a LVEDP above $16 \mathrm{mmHg}$ was only found in 8 out of 54 patients. As shown in Fig. 4, LVMDP was even more sensitive and specific in

$$
\begin{aligned}
& \text { - LV aneurysm } \\
& \text { : } E F \geqslant 50 \% \text { EF }<50 \%] \text { No LV aneurysm }
\end{aligned}
$$

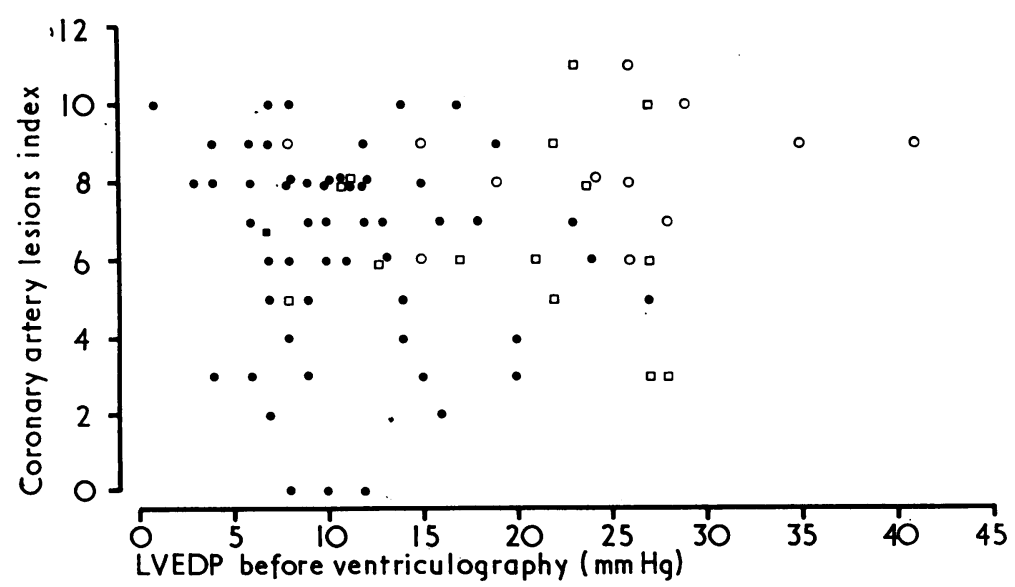

Fig. 3 Relation between LVEDP before ventriculography, and CALI. 


$$
\begin{aligned}
& \therefore \therefore \text { o } \\
& \text { - } \mathrm{EF} \geqslant 50 \% \text { [ }<0 \% \text { ] No LV aneurysm }
\end{aligned}
$$

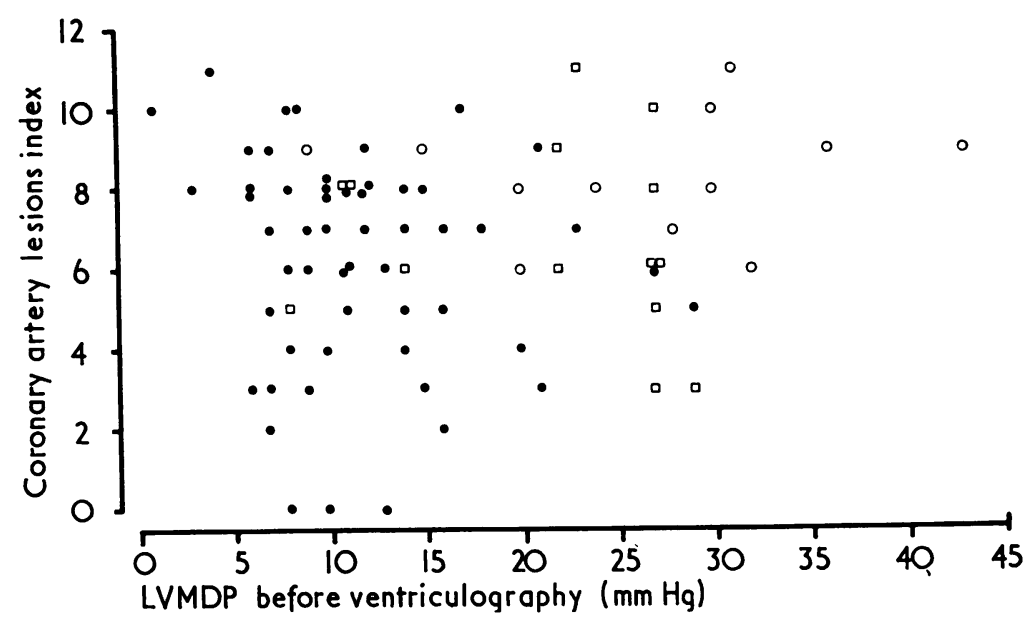

Fig. 4 Relation between LVMDP before ventriculography, and CALI.

this respect. LVMDP above $19 \mathrm{mmHg}$ was found in 20 out of the 26 patients belonging to either group 2 or 3 , as compared with only 6 out of the 54 patients in group 1.

After ventriculography no correlation between LVEDP and CALI was found (Fig. 5). The ability of LVEDP to predict dyskinesia was poorer after ventriculography. A positive and significant correlation between CALI, and $\triangle$ LVEDP and $\triangle L V M D P$ was found, but only in patients in whom LVEDP and LVMDP were normal before ventriculography (below $13 \mathrm{mmHg}$ ) (Fig. 6). Using $\triangle$ LVEDP the correlation coefficient was $0.51(n=41, P<0.001$, $95 \%$ confidence interval $0 \cdot 24$ to $0 \cdot 88$ ). The correlation coefficient using $\triangle$ LVMDP was $0.47(n=41$, $0.001<\mathrm{P}<0.01$ ). In Fig. 6 the patients are divided according to single, double, triple, and no vessel disease. $\triangle$ LVEDP above $12 \mathrm{mmHg}$ was found only in patients with triple vessel disease.

\section{RELATIONS BETWEEN ANGIOGRAPHY AND} DURATION OF SYMPTOMS

In our material CALI increased with the duration of the symptoms. In 25 patients with history of 2

$$
\begin{aligned}
& \text { - LV aneurysm } \\
& \text { - } E F \geqslant 50 \% \\
& \text { - EF<50\%] No LV aneurysm }
\end{aligned}
$$

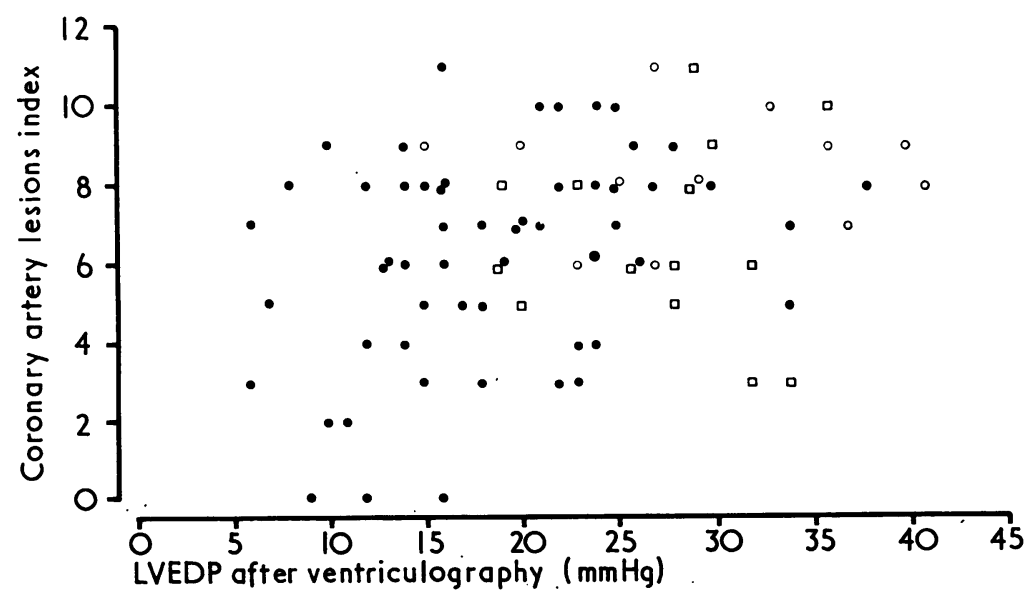

Fig. 5 Relation between LVEDP after ventriculography, and CALI. 


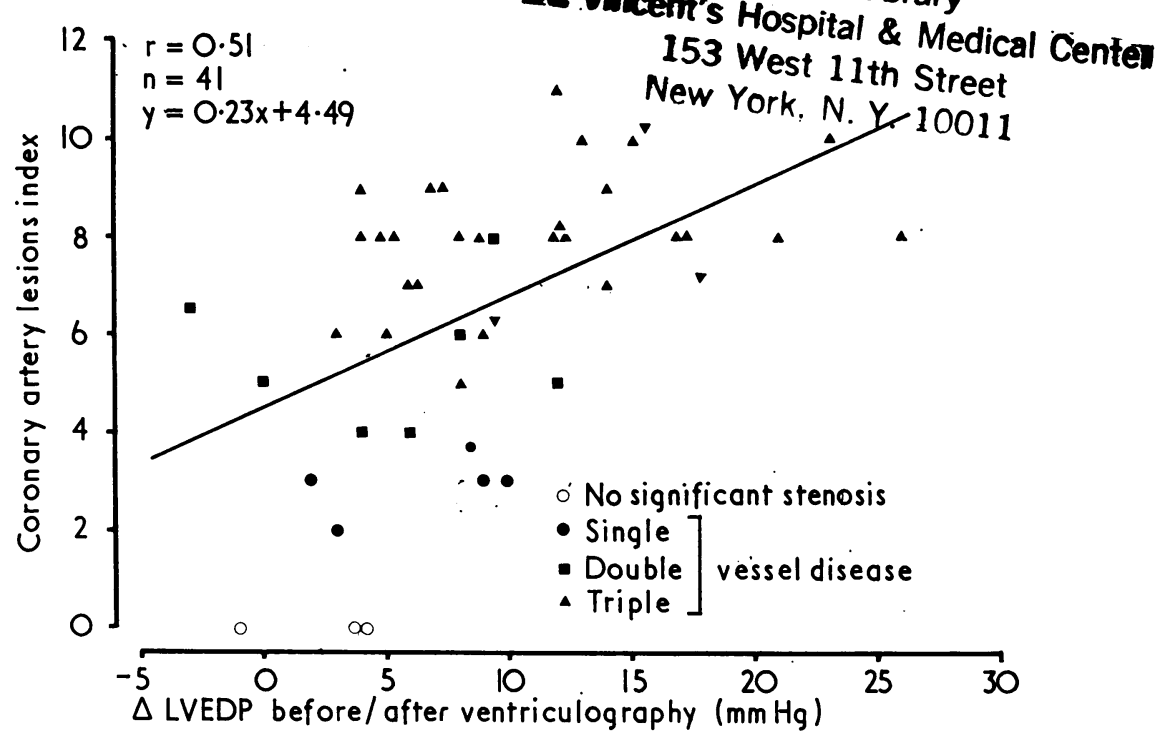

Fig. 6 Correlation between $\triangle L V E D P$ and CALI with differentation according to number of vessels affected.

years or less, mean CALI was 4.9 as compared with 7.4 in 55 with duration of more than 2 years $(P<0.001)$. Of these 55 patients 40 had triple, 11 double, and 4 single vessel disease.

In 7 patients with duration of 10 years or more, mean CALI was 8.3 and they all had triple vessel disease.

\section{Discussion}

In most of the patients with much reduced EF we found increased left ventricular filling pressure, both in those with angiographic appearances of left ventricular aneurysm and in those with akinesia without apparent aneurysm. The difference between aneurysm and akinesia is, however, difficult and unreliable, and some aneurysms may appear only as an akinetic area (Gorlin et al., 1967). The most important measure in this respect is considered to be the extent of the left ventricular wall without contraction. This is in accordance with the work of Klein et al. (1967) who by angiography and theoretical analysis found that when approximately 20 to 25 per cent of the left ventricular area is inactivated by any pathological process, the degree of shortening distance required of the myofibre to maintain stroke volume exceeds physiological limits, and cardiac enlargement (Starling mechanism) must ensue to maintain adequate ejection of blood. Such conditions are associated with increased end-diastolic volume and increased end-diastolic pressure.

The $a$ wave in the left ventricular pressure curve, which appears as a result of left atrial contraction, is prominent in such cases because of reduced ventricular compliance (Bristow et al., 1970; Smith et al., 1974). We found that the top of the $a$ wave (LVMDP) exceeded LVEDP (post a) more in patients with a raised filling pressure (LVEDP 15 to $30 \mathrm{mmHg}$ ) than in the others. We also found that LVMDP was slightly more reliable than LVEDP in the differentiation of patients with much reduced EF from the others, and $\triangle$ LVMDP correlated significantly with CALI, though less well than $\triangle$ LVEDP. LVMDP is often easier to determine than LVEDP. In cases with a prominent $a$ wave, the top of this wave is clearly defined. Determination of the true maximum diastolic pressure is difficult when the $a$ wave is continuous with the upstroke of left ventricular pressure curve. In these cases, however, the difficulties are the same whether LVEDP or LVMDP is measured.

$\mathrm{EF}$ is probably the most useful of the readily obtainable haemodynamic measurements in assessing the import of deranged left ventricular function in relation to the outlook for patients undergoing cardiac surgery (Cohn et al., 1974). LVMDP and LVEDP may reflect not only end-diastolic volume, but also wall stiffness, and is consequently not as indicative of contractile state as EF. This is possibly why a few cases with normal EF had considerable increase in LVMDP and LVEDP.

Injection of angiographic contrast medium into the left ventricle, the aortic root, or selectively into a coronary artery has immediate considerable haemodynamic effects, and an increase in the left ventricular filling pressure is a characteristic finding (Brown et al., 1969; Rahimtoola et al., 1970; 
Carleton, 1971; Kloster, et al., 1972; Cohn et al., 1973). The contrast medium unmasks latent dysfunction through its myocardial depressant effect as well as by augmentation of the Starling effect (Cohn et al., 1973).

Brundage and Cheitlin (1971) found that the increase in LVEDP after left ventricular angiography correlated with the degree of disease. Gensini et al. (1971) made similar observations after selective coronary arteriography. This is in agreement with the findings using exercise as a stress-test. Saltups et al. (1971) found that LVEDP during exercise was partly related to the distribution and severity of the coronary arterial lesions. We have found a significant correlation between the increments of LVEDP and LVMDP following left ventriculography and coronary arterial lesions (CALI in our study), in patients with normal LVEDP and LVMDP before ventriculography. This correlation probably arises from the relation between the reduction of left ventricular compliance and the degree of the coronary arterial lesions.

Even without preceding ventriculography a prominent $a$ wave is a frequent finding in the left ventricular pressure curve. Several workers have shown that reduced left ventricular compliance is an almost constant finding in coronary heart disease (Bristow et al., 1970; Diamond and Forrester, 1972). The reduced compliance leads to increased force of the left atrial contraction in order to achieve sufficient end-diastolic volume, and a prominent $a$ wave is the result. Therefore, the significance of the changes in LVMDP is reasonable. In coronary heart disease LVMDP seems to be as relevant a measurement as the traditional 'post $a$ ' LVEDP.

In cases with raised filling pressure before ventriculography no significant correlation was found between filling pressure increments and degree of coronary arterial lesions. A majority of these patients had previous myocardial infarcts, and dilated, noncompliant ventricles, suggesting an inability of enddiastolic fibre length to increase further.

In our material the distribution of coronary arterial lesions increased with the duration of the symptoms. This is to be expected in the light of the progressive nature of the arteriosclerotic process.

Left ventricular pressures before and after ventriculography are easily obtainable during routine left heart catheteriatsion. Studies of the diastolic pressures give valuable information about the functional state of the ventricle. If the diastolic pressure is normal before ventriculography, a large increase after ventriculography indicates serious coronary artery lesions. In our material all patients with LVMDP increment of more than $12 \mathrm{mmHg}$ had triple vessel disease, and 10 of 11 patients had very severe lesions (Fig. 6).

\section{References}

Arbogast, R., Solignac, A., and Bourassa, M. G. (1973). Influence of aortocoronary saphenous vein bypass surgery on left ventricular volumes and ejection fraction. Comparison before and one year after surgery in 51 patients. American Fournal of Medicine, 54, 290.

Bristow, J. D., Van Zee, B. E., and Judkins, M. P. (1970). Systolic and diastolic abnormalities of the left ventricle in coronary artery disease. Studies in patients with little or no enlargement of ventricular volume. Circulation, 42, 219.

Brown, A. K., Epstein, E. J., Coulshed, N., Clarke, J. M., and Doukas, N. G. (1969). Haemodynamic changes after angiocardiography. British Heart fournal, 31, 233.

Brundage, B. H., and Cheitlin, M. D. (1971). Left ventricular angiography as a function test. Circulation, 43-44, Suppl. II, p. 146.

Carleton, R. A. (1971). Change in left ventricular volume during angiocardiography. American fournal of Cardiology, 27, 460 .

Cohn, P. F., Gorlin, R., Cohn, L. H., and Collins, J. J., Jr. (1974). Left ventricular ejection fraction as a prognostic guide in surgical treatment of coronary and valvular heart disease. American fournal of Cardiology, 34, 136.

Cohn, P. F., Horn, H. R., Teichholz, L. E., Kreulen, T. H., Herman, M. V., and Gorlin, R. (1973). Effects of angiographic contrast medium on left ventricular function in coronary artery disease. American fournal of Cardiology, 32, 21.

Diamond, G., and Forrester, J. S. (1972). Effect of coronary artery disease and acute myocardial infarction on left ventricular compliance in man. Circulation, 45, 11.

Gensini, G. G., Dubiel, J., Huntington, P. P., and Kelly, A. E. (1971). Left ventricular end-diastolic pressure before and after coronary arteriography: the value of coronary arteriography as a stress test. American fournal of Cardio$\log y, 27,453$.

Goldschlager, N., Sakai, F. J., Cohn, K. E., and Selzer, A. (1970). Hemodynamic abnormalities in patients with coronary artery disease and their relationship to intermittent ischemic episodes. American Heart fournal, 80, 610.

Gorlin, R., Klein, M. D., and Sullivan, J. M. (1967). Prospective correlative study of ventricular aneurysm: mechanistic concept and clinical recognition. American fournal of Medicine, 42, 512.

Judkins, M. P. (1967). Selective coronary arteriography: Part I. A percutaneous transfemoral technic. Radiology, 89,815 .

Klein, M. D., Herman, M. V., and Gorlin, R. (1967). A hemodynamic study of left ventricular aneurysm. Circulation, 35, 614.

Kloster, F. E., Friesen, W. G., Green, G. S., and Judkins, M. P. (1972). Effects of coronary arteriography on myocardial blood flow. Circulation, 46, 438.

Rahimtoola, S. H., Gau, G. T., and Raphael, M. J. (1970). Cardiac performance after diagnostic coronary arteriography. Circulation, 41, 537.

Saltups, A., McCallister, B. D., Hallermann, F. J., Wallace, R. B., Smith, R. E., and Frye, R. L. (1971). Left ventricular hemodynamics in patients with coronary artery disease and in normal subjects: correlations with the extent of coronary artery lesions and the electrocardiogram. American Fournal of Medicine, 50, 8.

Smith, M., Russell, R. O., Jr., Moraski, R. E., Feild, B. J., and Rackley, C. E. (1974). Left ventricular A wave amplitude in patients after myocardial infarction. American fournal of Cardiology, 33, 370.

Requests for reprints to Dr. K. Forfang, Medical Department B, Rikshospitalet, Oslo 1, Norway. 